Andrea F. De Carlo

Dipartimento di Studi Letterari, Linguistici e Comparati dell' Università degli Studi di Napoli “L’Orientale"

\title{
Le lezioni sulla Divina Commedia di Józef Ignacy Kraszewski e gli inizi della dantistica polacca (1820-1870)
}

\section{La nascita della dantistica polacca}

Nella cospicua eredità artistica di Józef Ignacy Kraszewski (1812-1887), che fu principalmente caratterizzata dalla sua attività di romanziere, pubblicista e critico letterario, si può includere anche il suo contributo allo sviluppo degli studi danteschi in Polonia. Già il critico sovietico Ivan K. Gorskij annoverava lo scrittore tra i "fondatori” della dantistica polacca contemporanea [Gorskij 1968: 33]. Secondo l'esperto di letteratura polacca, il rapporto di Kraszewski con Dante si può riassumere in tre momenti essenziali: l'influenza di Dante sull'opera di Kraszewski [cfr. De Carlo 2016: 33-45; 2019a]; il contributo dello scrittore alla dantistica polacca con le lezioni del 1867, che tenne a Cracovia e Leopoli, pubblicate in seguito nel 1869 [cfr. Płaszczewska 2012: 191-203; 2017: 37-54; De Carlo 2019c: 363-381; 2019a]; infine la sua traduzione della Divina Commedia [cfr. De Carlo 2014: 291-309; 2017: 125-144; 2019b: 197-210]. .

1 Alcui frammenti o canti della Divina Commedia tradotti da Kraszewski sono pubblicati in Kraszewski 1866: 389-398; 1869a: 95-189; 1869b: 67-97; e ancora dei frammenti di Purg. XxxIII sono stati pubblicati in Dante 1870: 307-310; 1875: 127-131. Cfr. anche Miszalska et al. 2007; Płaszczewska 2012: 192; 2017: 39. 
In questa sede, tuttavia, ci soffermeremo ad analizzare solo le lezioni di Kraszewski sul poema dantesco, che furono date alle stampe con il titolo Dante. Studia nad Komedia Boska [Dante. Studi sulla Divina Commedia, 1869].

Nel momento in cui Kraszewski teneva le sue lezioni sulla Commedia, la Polonia aveva conosciuto da poco la pubblicazione dell'intero poema dantesco tradotto da Julian Korsak (1860) e la dantistica polacca muoveva ancora i suoi primi passi rispetto ad altri paesi come Francia, Inghilterra e la vicina Germania. ${ }^{2}$

I primi sviluppi della dantistica polacca infatti si possono far risalire al periodo compreso tra il 1820 e il 1835, allorché Kazimierz Brodziński (1791-1835) nelle sue dissertazioni di carattere critico-letterario spesso nomina Dante [Preisner 1957: 45-46]. Due anni dopo Dyzma Bończa Tomaszewski (1749-1825) pubblica una breve biografia del poeta italiano e sempre nello stesso anno il poeta Ludwik Osiński (1775-1838), professore di letteratura all'Università di Varsavia, sottolinea nelle sue lezioni l'originalità della Commedia [Preisner 1957: 45]. Con il proposito di divulgarne il nome e l'opera, l'Alighieri viene ricordato ancora da Maurycy Mochnacki (1803-1834) nei suoi articoli pubblicati tra il 1825 e il 1828 [Preisner 1957: 46]. Michał Wiszniewski (1794-1865), professore presso 1'Università Jagellonica, nelle sue lezioni sulla storia della letteratura, tenute negli anni 1831-1840, espone le sue meditazioni su Dante [Preisner 1957: 47]. Nel 1843 Dominik Chodźko (1796-1863) pubblica in Rimembranza il rifacimento di un lungo articolo sul divino poeta, che era apparso precedentemente sulla rivista russa “Severnaja Pčela” [Preisner 1957: 48]. Più tardi, nel 1845, Ludwik

2 L'amareggiato Kraszewski guardava proprio alla ricca dantistica tedesca che allora vantava gli studi di Karl Witte (1800-1883) o la traduzione artistica della Commedia del Philalethes (pseudonimo di re Giovanni di Sassonia, 1801-1873), data alle stampe nel 1867 . Negli studi danteschi re Giovanni poté contare, tra il 1839 e il 1853 , sulla competente collaborazione del suo bibliotecario e bibliografo Julius Petzholdt (1812-1891), autore di importanti bibliografie e studi su Dante. Se si tiene conto che fino al 1869 in Germania erano state già edite all'incirca - tra vecchie e nuove - venti traduzioni del divino poema, sono più che comprensibili le meste parole di Kraszewski annotate nelle sue lezioni [cfr. Kraszewski 1869a: 168]. 
Celiński fa stampare su "Przegląd Poznański" un articolo anonimo sulle fonti della Commedia [Litwornia 2005: 182].

Nel 1849 Władysław Sas Kulczycki pubblica sulla rivista "Athenaeum" di Vilnius (peraltro diretta tra il 1841 e il 1851 da Kraszewski) un saggio su Dante e la poesia italiana del Trecento. Questo studio critico fu accolto molto bene dagli ambienti letterari polacchi e consolidò il giudizio benevolo che egli, nonostante la sua giovane età, si era già guadagnato con la sua attività di scrittore $[\mathrm{cfr}$. Fiorentino 2003: 15].

Verso il 1850, quando il realismo trionfa nella letteratura e nelle scienze, la situazione nel campo della dantistica cambia radicalmente ovunque. Gli studiosi avviano in questo ambito tutta una serie di analisi scientifiche dei documenti, studi negli archivi e nelle biblioteche dove i dantisti si proponevano di scoprire nuovi materiali per poter decifrare sempre meglio la vita e le opere di Dante. In Italia i grandi maestri: Giosuè Carducci (1835-1907), Alessandro D’Ancona (1835-1914), Isidoro Del Lungo (1841-1927), Adolfo Bartoli (1833-1894) e molti altri possono essere considerati i veri fondatori della dantistica moderna italiana. La Francia cede il posto all'Inghilterra che viene degnamente rappresentata da Edward Moore (1838-1916) e Paget Toynbee (1855-1932). La Germania e anche la Svizzera continuano in modo eccellente gli studi danteschi avviati già sulla giusta strada da Witte e Philalethes, e a questi nomi andò ad aggiungersi una lunga lista di nuovi dantisti: Adolf Gaspary (1849-1892), Franz Xaver Kraus (1840-1901), Franz Xaver Wegele (1823-1897), Franz Hettinger (1819-189o) e soprattutto quello dello svizzero Giovanni Andrea Scartazzini (1837-1901). Ed è proprio qui che si deve collocare l'esordio della dantistica moderna polacca. All'opposto della Germania e dell'Italia che operavano come scuole di metodo, la Polonia non riuscì a fare di Dante un argomento d'interesse vasto e a fondare una scuola di studi danteschi. Certamente è da segnalare l'apprezzabile attività di certi studiosi, anche se hanno lavorato purtroppo isolatamente, senza un coordinamento delle ricerche e senza confrontare i risultati raggiunti [Morawski 1965: 90-91].

Verso la seconda metà del xIx e l'inizio del xx secolo il movimento di ricerca su Dante in terra polacca passa dalla sfera della 
letteratura, fondata principalmente sull'intuizione e la sensibilità estetica, a quella scientifica [Łempicki 1930: 39; cfr. altresì 193ob: 29-65]. In generale un momento particolarmente fecondo per lo sviluppo degli studi danteschi in Polonia risulta quello compreso tra il 1840 e il 1870 . Un contributo significativo alla dantistica polacca, dopo il periodo romantico, venne dato soprattutto dai lavori di Julian Klaczko, ${ }^{3}$ Julian Korsak, Józef Ignacy Kraszewski e Edward Porębowicz [cfr. De Carlo 2019a].

\section{Le lezioni su Dante}

È noto che nel 1851 Kraszewski aveva manifestato il desiderio di rivestire la carica di professore presso l'Università Jagellonica di Cracovia. Il piano delle lezioni avrebbe previsto inizialmente un intero semestre durante il quale egli avrebbe esposto, dopo un'ampia introduzione alla poesia cristiana medievale, le sue conferenze sulla vita e le opere di Dante, nonché sull'epoca in cui visse il poeta italiano [cfr. Kraszewski 1972 ${ }^{4}: 286 ; 292$ ].

Kraszewski tuttavia non riuscì a ottenere la cattedra di professore presso l'Ateneo cracoviense, ma in compenso nel $1867 \mathrm{fu}$ invitato dal Towarzystwo Wzajemnej Pomocy Uczniów Uniwer-

Klaczko (o Kłaczko) fu allievo delle università tedesche di Königsberg e di Heidelberg, dove ebbe modo di frequentare i corsi di Georg Gottfried Gervinus (1805-1871) sulla letteratura. Nel 1849 si trasferì a Parigi dove collaborò in modo più o meno regolare con le riviste "La Revue Contemporaine" e "La Revue de Paris", più tardi anche con "La Revue des deux mondes". Pubblicò un articolo su Heine e molti studi sulla letteratura francese e polacca, dando prova di grande preparazione scientifica e di acume critico [Morawski 1965: 91]. Gli studi danteschi di Klaczko cominciano con un lungo articolo in polacco: Dante Alighieri: I. Epoka Dantego II. Znaczenie Boskiej Komedyi (Dante Alighieri: I. L'epoca di Dante II. Il significato della Divina Commedia) che venne pubblicato nel 1852 sul IV ${ }^{\circ}$ volume di "Biblioteka Warszawska" (pp. 385-421). Dopodiché Klaczko diede alle stampe il suo studio critico Dante e la critique moderne apparso sulla "Revue Contemporaine" (11-15 novembre 1854) e tradotto in polacco come Dante wobec krytyki nowoczesnej in Klaczko 1904: 17-91. Nel 1860 Klaczko scrisse per la "Gazeta Codzienna" le famose Listy wtoskie (Le lettere italiane). Infine nel 1880 pubblicò la sua opera fondamentale: Causeries florentines: Dante et MichelAnge; Beatrice et la poésie amoureuse; Dante et le catholicisme; la tragédie de Dante. In seguito essa fu tradotta in polacco, in tedesco (1884) e in italiano: Klaczko 1925. 
sytetu Jagiellońskiego [Associazione di Mutuo Soccorso degli Allievi dell'Università Jagellonica] a tenere quattro conferenze pubbliche sulla Divina Commedia. Le dissertazioni di Kraszewski furono in seguito pubblicate sul "Rocznik Towarzystwa Przyjaciół Nauk w Poznaniu” nel 1869 con il titolo: Dante. Studia nad Komedia Boska. ${ }^{4}$ Nello stesso anno ne uscì un sunto nell'opera collettanea Kłosy $i$ kwiaty ${ }^{5}$ e, dopo un anno, le lezioni kraszewskiane furono tradotte in tedesco da Szczepan Bohdanowicz [cfr. Kraszewski 1870].

La critica considerò le lezioni di Kraszewski il più ampio e valido lavoro monografico sul vate fiorentino che fosse mai stato scritto sino a quel momento in lingua polacca [Biliński 1965: 16; Bachórz 1990: 154; Litwornia 2005: 193-197; Płaszczewska 2012: 192; 2017: 39]. Il contenuto fu accolto come una buona introduzione alla lettura del poema, in cui l'autore presentava lo sfondo storico dell'epoca, introducendo e analizzando la figura di Dante come uomo, politico e poeta geniale. Per l'epoca Kraszewski dimostrò una profonda conoscenza dell'autore e della sua opera sia nel descrivere in maniera minuziosa la vita del vate fiorentino sia nel fornire un'analisi puntuale della Commedia [cfr. Bursztyńska 1998: 45], anche se - come vedremo più avanti - alcune ipotesi da lui formulate sono ormai ampiamente confutate dalla critica.

Le conferenze di Kraszewski si possono includere in quel profluvio di eventi europei che si ebbe a partire dal 1865 in occasione del secentenario della nascita di Dante. La città di Cracovia, di conseguenza, nel 1867 volle onorare con la voce di Kraszewski, quale espressione del rapporto che legava i polacchi al poeta fiorentino, l'anniversario dantesco. La pubblicazione di queste lezioni media, letti a Cracovia ed a Lepoli nel 1867. Domenico Ciampoli nel suo articolo su "Fanfulla della domenica" (n. 15, 10 aprile 1887), in seguito incluso in Studi letterari (Catania 1891, pp. 401-415), lamenta che fino ad allora non vi erano traduzioni in italiano delle opere di Kraszewski, eccetto una traduzione italiana degli studi su Dante rimasta inedita che sarebbe stata redatta da una contessa di cui l'autore non rivela il nome. Lo studioso italiano riferisce: "Molte [... ] opere sono tradotte in tedesco, alcune in francese, quasi nessuna in italiano; gli studi su Dante solo, e sono disgraziatamente inediti, dalla signora contessa" [Ciampoli 1891: 410].

5 In Kłosy i kwiaty vi è solo un frammento della traduzione kraszewskiana: Raj XVII, vv. 55-69 [Kraszewski 1869b: 88-89]. 
andava ad aggiungersi ai numerosi resoconti che erano apparsi sulla stampa europea; inoltre esse avevano come intento precipuo quello di rendere più familiare ai polacchi la Commedia e il suo autore.

Le informazioni sul soggiorno di Kraszewski a Cracovia e sulle sue conferenze furono pubblicate il 25 aprile 1867 sulla rivista "Czas" e sulla "Gazeta Polska” di Varsavia; in quest'ultima l'articolo fu corredato di informazioni bibliografiche su Dante a cura di Karol Estreicher ${ }^{6}$. Più tardi un ampio e puntuale resoconto su come si svolsero i fatti fu dato da Michał Frąckiewicz nel suo saggio edito a Cracovia nel 1912: Józef Ignacy Kraszewski w Krakowie i we Lwowie w r. 1867 i odczyty jego o Dantem. Wspomnienia z przeszłości w setna rocznicę urodzin [Józef Ignacy Kraszewski a Cracovia e a Leopoli nel 1867 e le sue lezioni su Dante. Ricordi del passato nel centenario della nascita].

Il soggiorno di Kraszewski nella città di Cracovia durò dal 27 aprile al 7 maggio 1867: durante questi dieci giorni lo scrittore riuscì a dedicarsi ai suoi progetti e visitò la Biblioteca Jagellonica, dove raccolse materiale per la sua opera in tre volumi Polska $w$ czasie trzech rozbiorów 1772-1799 r. Studia do historyi ducha i obyczaju [La Polonia ai tempi delle tre spartizioni 1772-1799. Studi sulla storia dello spirito e delle tradizioni, Frąckiewicz 1912: 12].

Come era consuetudine a quei tempi, le conferenze si svolsero nell'edificio dell'Associazione delle Scienze e delle Lettere in via Sławkowska - oggi edificio della Polska Akademia Umiejętności (PAU) - il 29 aprile e si susseguirono nei giorni 1, 4 e 6 maggio. Per tutte e quattro le lezioni il pubblico fu alquanto numeroso. Esse suscitarono un notevole interesse nonostante il nome di Dante fosse pressoché sconosciuto.?

6 Il titolo dell'articolo, che uscì su "Gazeta Polska" (n. 9o, il 18 aprile 1867), era Przektady Danta [Frąckiewicz 1912: 11].

7 Kraszewski destinò i profitti delle sue conferenze di Cracovia al Towarzystwo Wzajemnej Pomocy Uczniów Uniwersytetu Jagiellońskiego (cfr. Burkot [nota 6], in Kraszewski 1993: 53. In una lettera inviata da Dresda al fratello Kajetan il giorno 12 aprile 1867, lo scrittore scrive: "W Krakowie i Lwowie będę miał na korzyść ubogich odczyty publiczne o Dancie. I to koszt, ale na ten narazić się i biednemu trzeba, gdy idzie o biedniejszych jeszcze, a mających nadzieje życia przed sobą”, in Kraszewski 1993: 52 ("A Cracovia e Leopoli avrò alcune conferenze su Dante a beneficio dei poveri. È un costo, ma a esso si deve esporre anche il povero, 
Nel suo resoconto, Frąckiewicz riferisce dettagliatamente come si svolsero gli incontri: ${ }^{8}$ la prima lezione si tenne il 29 aprile 1867 e riguardò un'introduzione generale del poema; Kraszewski fece una presentazione della vita di Dante e alcune osservazioni generali sulla sua formazione, la singolarità del suo genio e l'esilio. La seconda conferenza ebbe luogo mercoledì $1^{\circ}$ maggio: l'autore espose la parte finale della vita di Dante, citando biografi e commentatori del divino poeta; dopodiché passò a presentare la struttura del poema. Nel terzo incontro, che si tenne il 4 maggio, Kraszewski fece un sunto dettagliato del contenuto della Commedia, in particolare del Purgatorio; egli mise in evidenza alcuni episodi della seconda cantica citando numerosi frammenti della sua traduzione [Frąckiewicz 1912: 38]. Infine, lunedì 6 maggio 1867 si svolse la quarta e ultima conferenza, dove l'autore propose una sintesi e un'analisi breve del Paradiso e concluse con la lettura di ampi frammenti del poema [Frąckiewicz 1912: 44].

La sera dello stesso giorno Kraszewski partì per Leopoli, dove giunse il giorno successivo. Qui l’autore di Stara baśń (La favola antica, 1876) era stato invitato dall'organizzazione patriottica studentesca conosciuta col nome di "Czytelnia Akademicka", che si era costituita nel 1867 su modello di quella nata precedentemente presso l'Università Jagellonica. La vita dei giovani studenti di Leopoli era in questo periodo fortemente germanizzata, il che suscitò un rinvigorimento della lingua e della letteratura nazionali.

A Leopoli lo scrittore restò una decina di giorni e tenne le sue lezioni nella sala del consiglio cittadino rispettivamente l'8, 11, 12 e 15 maggio. ${ }^{9}$ Qui Kraszewski replicò quanto esposto in precedenza a Cracovia, con la differenza che a Leopoli vi fu un'esigua

quando si tratta soprattutto dei più poveri, che hanno speranze di vita davanti a sé"). [Qui e successivamente, le traduzioni dal polacco, dove non diversamente indicato, sono mie - ADC].

8 Frąckiewicz propone un'ampia sintesi delle lezioni di Kraszewski; occorre, inoltre, precisare che i frammenti riportati dal critico sono attinti dal saggio Dante. Studia nad Komedia Boska, ma in essi si riscontrano refusi e persino omissioni di interi versi.

9 I profitti delle conferenze di Leopoli furono destinati alla "Czytelnia Akademicka”. Kraszewski qui ottenne la cittadinanza onoraria della città e in teatro fu rappresentata la sua commedia Panie Kochanku: anegdota dramatyczna w 3 aktach 
partecipazione di pubblico. Questo fatto, molto probabilmente, fu conseguenza della pubblicazione nello stesso anno dei Rachunki (Resoconti), ${ }^{10}$ che suscitarono un diffuso sentimento di acrimonia nei confronti di Kraszewski. Ciononostante, l'illustre romanziere e le sue conferenze su Dante furono accolti con grande entusiasmo [Frąckiewicz 1912: 53] come si desume da un articolo del 14 maggio 1867 apparso su "Dziennik Literacki", in cui il redattore, Juliusz Starkel, salutò con viva cordialità e profondo ossequio la presenza di Kraszewski a Leopoli [Frąckiewicz 1912: 51]..1

\section{Kraszewski esegeta di Dante}

Gli Studia nad Komedia Boską sono costituiti da un'introduzione, un'analisi tematico-contenutistica e una conclusione. Nell'esordio, l'autore presenta alcuni frammenti della vita di Dante in cui vi delinea la personalità del genio italiano, la sensibilità poetica, nonché gli eventi più importanti che hanno contraddistinto la sua

(Signor Amante! Un aneddoto drammatico in tre atti, 1867; cfr. Burkot, [nota 6], in Kraszewski 1993: 53).

Pubblicati dall'autore tra il 1866 e il 1870, in essi l'autore mise in luce tutti i fenomeni della vita culturale, politica e sociale del proprio paese, chiamando alla resa dei conti, per l'appunto, la nobiltà terriera da cui egli stesso proveniva: attaccò l'alleanza clerico-feudale, smascherò gli intrighi e combatté gli ultramontani [cfr. Biliński 1971: 25].

11 Ciò è attestato anche dallo stesso Kraszewski in una lettera che inviò il 15 maggio 1867 a suo fratello Kajetan: "Kochany Kajetanie! Wierz lub nie wierz, że chwilowo jestem w Galicji. Przybyłem do Krakowa na wezwanie akademików, aby dla ubogich odczytami zapewnić jakąś pomoc. O to samo proszony ze Lwowa, nie mogłem odmówić. Dziś mój ostatni tu odczyt o Dancie na korzyść Czytelni Akademickiej. Przyjęto mnie i w Krakowie, i we Lwowie nader i do zbytku serdecznie. Obiady, wieczory, fety etc. niustające. Mieszczanie zbierają się dziś dać mi dyplom na honorowe obywatelstwo, co dotąd tylko ministrom i namiestnikom się dawało.", Kraszewski 1993: 55 ("Amato Kajetan! Non ci crederai, ma al momento mi trovo in Galizia. Sono stato a Cracovia su invito degli accademici, per fornire ai meno abbienti un aiuto con alcune conferenze. Lo stesso mi è stato chiesto a Leopoli, e non potevo rifiutare. Oggi qui è la mia ultima conferenza in favore della "Czytelnia Akademicka". A Cracovia e a Leopoli sono stato accolto fin troppo calorosamente. Incessanti pranzi, serate, feste ecc. Oggi i cittadini si raduneranno per consegnarmi il diploma di cittadinanza onoraria, che finora è stato dato solo a ministri e governatori."). 
esistenza: l'amore, la politica e l'esilio. Qui Kraszewski formula la tesi sull'eccezionalità di Dante, che in seguito dimostrerà e motiverà nel corso dell'esposizione. A questa presentazione generale segue la parte principale dell'elaborato che consiste in un ampio sunto della Divina Commedia, congiuntamente a un'approfondita disamina testuale di determinati canti ed episodi del poema [cfr. Płaszczewska 2012: 196; 2017: 44-45]. Infine, l'autore nelle conclusioni si concentra principalmente sulla ricezione di Dante nel più ampio contesto europeo e in quello prettamente polacco [cfr. altresì Płaszczewska 2012: 195-196; 2017: 43-44].2

Kraszewski considera Dante piuttosto un realista, ma con la sensibilità rinascimentale, e con l'attitudine a rappresentare incertezze e vive impressioni servendosi della sua fervida immaginazione. Lo scrittore polacco, nelle sue lezioni, colloca le origini della rinascita culturale europea nel $\mathrm{X}$ secolo, non appena gli influssi bizantini e arabi si erano fatti sentire in Europa. Questa conduce al risveglio dell'autocoscienza nazionale, alla comparsa dei primi

Come giustamente osserva Olga Płaszczewska, le lezioni di Kraszewski presentano una struttura chiusa. Lo studioso ucraino Volodimir Vasilenko fa un confronto tra la tecnica narrativa dello scrittore polacco e quella di Dante [Płaszczewska 2017: 44; 2012: 196]: "po mistrzowsku włada metodą modelowania tego, co ogólne, rysując wciąż nowe płaszczyzny semantyczne - wzajemnie dopełniające się w zwrotach, skrzyżowaniach i nagromadzeniach, kiedy to raz po raz zmieniający się punkt widzenia pozwala nie tylko uniknąć monotonii w rozwoju przedmiotu, ale i osiągnąć obraz stereoskopowy w czasie jego prezentacji [Wasyłenko 1993: 19-20]. ("È in grado di modellare magistralmente ciò che è generale, disegnando costantemente nuovi piani semantici, che si completano a vicenda in frasi, intersezioni e accumulazioni, mentre il continuo cambio di punto di vista permette non solo di evitare uno sviluppo monotono dell'argomento, ma anche di ottenere nel corso della sua presentazione un'immagine stereoscopica"). Inoltre, Płaszczewska nota che nel saggio di Kraszewski il confronto del poema di Dante con la letteratura del XIX secolo si sviluppa su due binari. Il primo è un raffronto delle caratteristiche della lingua di Dante con le correnti contemporanee. Il secondo raffronta due autorità, due rappresentanti di prima grandezza della letteratura medievale e romantica: Dante e Mickiewicz. Facendo un confronto del testo originale di Ugolino con la traduzione di Mickiewicz, Kraszewski afferma che si tratta di una "geniale parafrasi" del testo di partenza. L'idioletto ottocentesco di Mickiewicz non è più quello medievale di Dante, in quanto l'epoca di Mickiewcz è diversa da quella in cui visse Dante, così come dissimili sono i suoi destinatari [Płaszczewska 2017: 47; 2012: 198]. 
documenti in lingua nazionale in Francia, Germania, Inghilterra, Scandinavia e in altri paesi europei [Gorskij 1968: 39]. Infatti lo sviluppo culturale, che raggiunge il suo apogeo nel XIII secolo, si differenziò per carattere e importanza a seconda delle nazioni [Morawski 1965: 94-95].

L'autore, dopo aver presentato le caratteristiche ricorrenti in tutte le opere di Dante, si sofferma brevemente sulla Vita nuova. Qui Kraszewski, come Klaczko, propende per la reale esistenza di Beatrice [Morawski 1965: 95]: essenzialmente non accettava l'opinione di coloro che l'avevano considerata solo una pura allegoria, un mero frutto della fantasia dantesca. Beatrice, a detta di Kraszewski, divenne principalmente la musa ispiratrice del poeta [Gorskij 1968: 40] e nella sua vita giocò il ruolo "tego drugiego mistrza, co po Brunettim nauczyć go miał cierpiéć, wierzyć, żyć światłem, duchem, niebem" [Kraszewski 1869a: 105]. ${ }^{13}$ Dopodiché l'autore passa ad analizzare la visione stilnovistica della donna: i poeti avevano smesso di guardare ad essa come una "baccante umiliata" ("upodlona bachantka") [Kraszewski 1869a: 106], dal momento che la passione carnale si era trasformata in sentimento trascendente. La donna era contemplata non più solo per la sua bellezza materiale, ma in quanto strumento di elevazione spirituale. ${ }^{14}$ Kraszewski attribuisce questa "deviazione" della cultura al notevole influsso che il Cristianesimo rivestì nel mondo medievale; inoltre egli coglie un nesso tra la donna angelicata e il culto della Santa Vergine.

Lo scrittore, nel contempo, analizza l'artificio dell'“autobiografia” descritta nella Commedia, ossia un racconto letterario narrato dal medesimo Dante, al fine di esporre e affrontare nell'opera i mali del tempo. L'elemento biografico del poeta doveva servire sia a far recepire il viaggio nell'aldilà come reale sia a evidenziare il genio trasposto nella materia poetica. Kraszewski rifiuta il modello positivista tipico del ritratto psicologico ed esamina il poema prediligendo piuttosto un'esposizione romanzata con frequenti riferimenti alla biografia di Dante. Il mondo dell'oltretomba, come luogo di espevivere colla luce, lo spirito, il cielo".

14 Kraszewski rilegge l'incontro di Dante e Beatrice quale allegoria di penitenza e ammissione delle proprie colpe [Płaszczewska 2012: 199]. 
rienze metafisiche di un artista-intellettuale, rappresenta in verità un ritorno al mondo reale. Nella Commedia il poeta riveste due ruoli: quello di narratore che a distanza di tempo analizza il suo viaggio nell'aldilà, e quello di poeta-personaggio, principale figura del poema, che attraversa il mondo metafisico e sperimenta i sentimenti di dolore e di privazione [Bursztyńska 1998: 46]. Kraszewski reputa alquanto singolari le esperienze e i sentimenti che Dante conosce e prova nei tre regni oltremondani: "Interes główny skupia się koło Boga i człowieka; to są dramatis personae, główne; reszta episodyczna i podrzędna" [Kraszewski 1869a: 127]; "tu bohaterem jest nie pół-bóg, wódz, ani król, ani gród lub państwo, jest nim sam poeta, mylę się, - jest nim człowiek" [Kraszewski 1869a: 126]. ${ }^{15}$ Dante, dunque, esprime nelle sue preoccupazioni il sentimento di tutta l'umanità che è la vera protagonista del dramma e su di essa e i suoi destini verte la Commedia. Le sorti del genere umano costituiscono l'unità dell'azione: "Ludzkość, jéj przeznaczenie, uosobione są w Dancie, pojęte wedle idei chrześcijańskiéj” [Kraszewski 1869a: 126]. ${ }^{16}$ Kraszewski legittima l'umanesimo di Dante essenzialmente come espressione dell'etica cristiana: il vate non presenta se stesso come eroe ma come creatura imperfetta, peccatrice e debole, che riconosce i propri errori, sofferente e continuamente corrotta dal peccato [Kraszewski 1869a: 126].

L'autore polacco, nel narrare uno degli episodi più commoventi della Divina Commedia, ovverosia la storia di Paolo e Francesca, palesa il pathos umano di Dante: "Zaprawdę, możeż być co bardziéj ludzkiego nad tę litość, którą poeta nie jeden raz okazuje w piekle dla potępionych? Nie złorzeczy on przeciwko wyrokowi Bożemu, czuje go sprawiedliwym, ale los tych dusz go przejmuje - aż do zmysłów utraty" [Kraszewski 1869a: 137]. ${ }^{.7}$ Anche il lessico del

15 "Il principale interesse si concentra su Dio e sull'uomo; queste sono le dramatis personae, le più importanti; il resto è episodico e secondario"; "qui il protagonista non è un semidio, un condottiero, né un re né una città o uno Stato, è il poeta stesso, anzi, è l'uomo".

16 "L'umanità, il suo fato, sono rappresentati in Dante, recepiti secondo l'idea cristiana”.

17 "Forse che ci può essere qualcosa di più umano della compassione che il poeta sovente manifesta verso i dannati dell'inferno? Egli non inveisce contro le sen- 
poema appare molto più naturale nel momento in cui egli descrive i drammi umani:

Dante często w kwestijach teologicznych i filozoficznych, gdy mu przychodzi ująć w słowa temat czysto rozumowy, obyczajem wieku igra z wyrazy, dobiera je, bawi się niemi, układa je mozajkowo, nadaje im znaczenia różne, wykwintne; ale gdy maluje tragiczne losy człowieka, jego namiętności, niedolę, słabości, jest natchnionym malarzem, tworzącym obrazy, które na wieki wypiętnowane zostają w umyśle tych co je raz mieli przed sobą [Kraszewski 1869a: 137]. ${ }^{18}$

Secondo Kraszewski, il Cristianesimo ha determinato lo sviluppo storico-culturale e plasmato quella forza interiore che è insita nelle tradizioni e nei popoli di ogni epoca. L'autore, dunque, si avvicinò alla Commedia con la sua concezione idealistica della storia, che postula la vittoria sicura dello spirito sulle forze materiali. Nelle lezioni su Dante vi è già il germe di quella visione storica che più tardi lo scrittore tratterà più diffusamente nel suo lavoro, già citato, Polska w czasie trzech rozbiorów 1772-1799 $r$.

Nella tragica realtà polacca il poema dantesco ha significato per molti il tentativo di salvare se stessi dalla disperazione, di trovare un valido espediente di evasione di fronte alla triste situazione in cui la Polonia era caduta dopo la sconfitta dell'insurrezione del 1863 [Gorskij 1968: 46]. Kraszewski, a un certo punto, introduce il tema dell'esilio: egli narra le vicissitudini di Dante e polemizza con quei commentatori che hanno congetturato nel poema un sentimento di vendetta nei confronti di Firenze:

tenze di Dio, lo sente giusto, ma il destino di quelle anime lo coinvolge fino alla perdita di coscienza".

"Dante spesso nelle questioni teologiche e filosofiche, quando vuole esprimere a parole un argomento puramente razionale, come era consuetudine all'epoca, gioca con le parole, le sceglie, si diletta con esse, le dispone a mosaico, conferisce loro un significato nuovo, elegante; ma nel momento in cui effigia i destini tragici dell'uomo, la sua passionalità, la sventura, la debolezza, è un pittore ispirato e crea immagini che restano nei secoli impresse nella mente di coloro che almeno una volta le hanno avute di fronte". 
W początkach, jak każdy tułacz, Dante nie przypuszczal, ażeby powrót do ojczyzny miał mu być na wieki wzbronionym. Wraz $z$ innemi skazanemi na wygnanie pisał on listy błagające przebaczenia, domagające się raczéj usprawiedliwienia i powrotu, przyrzekając wstrzymać się od wszelkich kroków nieprzyjacielskich względem niechętnych, od wszelkich knowań przeciw zwycięzkiemu stronnictwu" [Kraszewski 1869a: 113].19

L’autore di Stara baśń, al contrario, discerne proprio nelle parole di Cacciaguida il patriottismo di Dante:

Tu proverai sí come sa di sale

lo pane altrui, e come è duro calle

lo scendere e 'l salir per l'altrui scale.

(Par. XviI, 58-60)

Da questo e da molti altri frammenti del poema, in cui traspare chiaramente l'ardore dell'esule, lo scrittore polacco arguisce la chiave di lettura per capire l'amore sincero del poeta verso la patria; ma questo amore fa presagire a Dante l'infelice prospettiva della caduta di Firenze.

\section{Il valore della Commedia}

Kraszewski, in seguito, affronta la questione della lingua, affermando che proprio su questo piano si esplica l'originalità di Dante; il dialetto toscano nobilitato al rango di lingua letteraria diventa lo strumento privilegiato dell'enunciazione poetica:

Język poematu, który Dante wziąl prawie surowym z ust ludu i do tak wielkiéj doskonałości wykształcił, iż po dziś dzień stylem mu nie zrównał nikt, brzmi jak melodija najwdzięczniejessergli per sempre proibito. Insieme con gli altri condannati all'esilio egli scrisse lettere che imploravano perdono, che anzi chiedevano la discolpa e il ritorno, promettendo di astenersi da qualsiasi azione nemica verso gli avversari, da qualunque macchinazione contro il partito vincitore." 
sza. Dante pierwszy nadał téj mowie włoskiéj, językowi dotąd jeszcze nieustalonemu, piśmiennie ledwie wschodzącemu w życie - stalsze formy, przez które daléj się miał kształcić [Kraszewski 1869a: 186]. ${ }^{20}$

Lo scrittore individuò il valore della Divina Commedia anche nei suoi aspetti etico-morali. L'esperienza dantesca mostra essenzialmente l'impellenza di una riforma morale nel mondo dei vivi: il poeta è un uomo reale, un Odisseo del Medioevo cristiano, che attraversa i tre regni oltremondani per indicare all'umanità la strada verso la salvezza. Kraszewski, oltre a dare una rilettura del viaggio metaforico di Dante con il prisma della realtà contingente, dimostrò come il grande poeta italiano si fosse ispirato ai miti antichi (basti qui ricordare, a titolo di esempio, la Visione di Tundalo), che raccontavano di viaggi nell'aldilà o in paesi misteriosi e lontani. L'ispirazione medievale non escludeva la profonda ammirazione per la cultura antica il cui rappresentante più eminente fu Virgilio. Kraszewski approfondisce i rapporti tra i due poeti e mette in luce l'idea originale che Dante si era fatto del poeta dell'Eneide; la sua visione del poeta latino era del tutto indipendente dalle leggende che circolavano nel Medioevo [Morawski 1965: 95].

Lo scrittore polacco, presentando l'attività politica dell'Alighieri, polemizza con Cesare Balbo (1789-1853) e tutti coloro che tacciarono il poeta fiorentino di versatilità: "okazał się wyższym nad swój wiek i spółczesnych. Nie był ani gwelfem ani gibellinem, czarnym ani białym, był wiernem dziecięciem Florencyi, kochającym kraj, pragnącym dlań zgody i spokoju” [Kraszewski 1869a: 109]. ${ }^{21}$ L'autore degli Studia vede in questo atteggiamento la dimostrazione dello spirito nobile e indipendente del poeta fiorentino, poiché

"La lingua del poema che Dante attinse pressoché acerba dalle labbra del popolo e potenziò fino a una così grande perfezione, che sino a oggi nessuno lo ha eguagliato nello stile, risuona come melodia graziosissima. Dante per primo assegnò al volgare italiano, una lingua fino ad allora ancora non consolidata, alla scrittura che a fatica si affermava nella quotidianità - forme fisse che in seguito dovette sviluppare." "risultò il più grande del suo secolo e tra i suoi contemporanei. Non fu né guelfo né ghibellino, né nero né bianco, fu un fedele figlio di Firenze, che amava il paese e desiderava per esso armonia e pace." 
egli cercò la salvezza di Firenze e la trovò: "nie w ludziach i stronnictwach, ale w zasadach i ideach" [Kraszewski 1869a: 110]. ${ }^{22}$ Inoltre, se Dante non avesse preso parte alle lotte politiche - a cui Kraszewski assegna un grandissimo significato storico - non sarebbe mai nata la Divina Commedia [Gorskij 1968: 41].

\section{Gli Studia e la critica del tempo}

Nelle lezioni di Kraszewski non mancano certo prese di posizione alquanto controverse: a titolo di esempio si può citare il soggiorno di Dante a Parigi e a Oxford che lo scrittore considera come eventi certi. Se il viaggio in Francia era ritenuto da molti dantisti moderni come probabile, tutti o quasi tutti, invece, reputavano il presunto periodo di studi in Inghilterra inverosimile. Kraszewski, convinto della sua idea, analizza le relazioni di Dante con la cultura inglese e accetta come sicuro l'influsso di Ruggero Bacone sull'autore della Commedia ${ }^{23}$. Pur rendendosi conto delle differenze esistenti fra i due autori, egli resta un convinto assertore dell'influenza dello scienziato inglese su Dante che, a suo parere, "powtarza Bacona prawie z tąż samą gwałtownością, gniewem, oburzeniem” [Kraszewski 1869a: 116]. ${ }^{24}$ Secondo l'autore, i commentatori del vate fiorentino hanno sempre sottovalutato la possibilità di un soggiorno del poeta ad Oxford e delle conseguenze che esso avrebbe potuto determinare. È verosimile che qui Dante avesse conosciuto il manoscritto dell' Opus Majus, la più famosa opera scientifica del tempo, e che avesse dunque attinto a essa durante la stesura della Commedia [Kraszewski 1869a: 116]. Scartazzini criticò duramente Kraszewski per questa sua convinzione:

che Dante viaggiò a Oxford e che i Malaspina gli fornirono i mezzi onde fare il viaggio; che il poeta attinse tutta quanta del pensiero di Ruggero Bacone su Dante, dal momento che l'Opus Majus era ritenuta l'opera scientifica più importante dell'epoca da cui il poeta avrebbe potuto attingere teorie di scienze ottiche e non solo [cfr. Finazzi 2008: 190]. "si rifà a Bacone quasi con la stessa veemenza, ira, indignazione". 
la sua scienza dall'Opus Majus di Ruggero Bacone. Il valore di queste e di infinite altre scoperte di tal natura si può facilmente indovinarlo" [Scartazzini 1871: 524-526; oppure cfr. Scartazzini 1881: 203-205].25

Inoltre il dantista svizzero biasimò molte altre informazioni ritenute non storicamente attendibili, come l'esistenza reale di alcuni personaggi, nonché per gli errori di stampa e la debole traduzione in tedesco.

Le difese di Kraszewski furono prese, molti anni dopo da un altro critico non dantista ma conoscitore delle letterature slave, Domenico Ciampoli; il suo giudizio fu diametralmente opposto a quello di Scartazzini, soprattutto rivalutando Kraszewski come scrittore [Piekut 1967: 36]:

Nel romanzo, nella novella Kraszewski fece parte di se stesso; fu un romantico che sta a disagio tra le partizioni scolastiche, perché non imitò alcuno, o se imitò, imitò il popolo di quella imitazione che ricrea. La spiccata umanità dei suoi personaggi ricorda il nostro Manzoni o lo Scott... [Ciampoli 1891: 41 ${ }^{26}$.

Nel saggio di Kraszewski vi sono molte altre prese di posizione che ormai la dantistica considera superate: ancora a titolo di esempio si può menzionare la leggenda dell' infelice matrimonio di Dante, in cui l'autore si attiene alla versione di Boccaccio senza sottometterla a un esame critico. Però vi sono anche valide intuialla versione tedesca, talvolta troppo letterale, a opera di Bohdanowicz. Il giudizio caustico di Scartazzini, pubblicato inizialmente in appendice sul quotidiano “Augsburger allgemeine Zeitung" del 1870 (nn. 217-218) e l'anno successivo rieditato su "Nuova Antologia", vol. XVII, è ripreso da Giuseppe Jacopo Ferrazzi nella sua Enciclopedia dantesca (vol. 4, Tipografia Sante Pozzato, Bassano 1871), in cui scrive a pag. 19: "Il lavoro è diviso in due parti: la $\mathrm{I}^{\mathrm{a}}$, poco erudita e non iscevra di errori, parla dei tempi e della vita di Dante; la $\mathrm{II}^{\mathrm{a}}$ è un'analisi in prosa, e troppo superficiale della Divina Commedia". I riferimenti a Scartazzini sono indicati dal medesimo Ferrazzi a pag. 241; cfr. anche i commenti di Claudio Mariotti in Boito 2008: 97. lo stesso articolo è stato pubblicato su "Fanfulla della domenica", n. 15, 1887. 
zioni che precedono quelle attuali: per esempio, l'opinione confutabile dell'influsso di Dante sull'arte del Trecento, in particolare su Giotto e i suoi affreschi nella Cappella degli Scrovegni a Padova. Secondo Kraszewski, ingiustamente si ascrive a Dante l'idea degli affreschi giottiani [cfr. Płaszczewska 2017: 50-51; 2012: 200-201]:

W Boskiej komedii Dantego jeniusz poety złożył w potężną całość to, co naówczas żyło w tradycjach ludów i kościoła, Giotto i Dante czerpali z jednego źródła, nie zapożyczając się od siebie, biorąc ze skarbnicy ogólnej. Stąd naturalnie i podobieństwo ich obu [Kraszewski 1866a: 180-181 ${ }^{27}$.

Nelle lezioni non mancarono pareri strettamente personali del dantista polacco, e tra esse si deve annoverare l'opinione secondo cui la seconda cantica detenesse il primato di bellezza [cfr. Płaszczewska 2017: 48; 2012: 199]. Kraszewski riteneva il Purgatorio la cantica più appassionante e meritevole di ammirazione di tutta la Commedia: in essa - come l'autore arguisce - il poeta raccolse tutta l'immensità sia del suo profondo scibile che della sua fervida immaginazione. Se nell' Inferno il poeta s' ispira alle leggende, ai miti e alle tradizioni, nel Purgatorio s'incontra l'uomo nella sua immagine più terrena, che si purifica e si nobilita da tutti i peccati e gli errori commessi in vita. La seconda cantica inoltre - secondo Kraszewski - dovrebbe convincere che Dante non fu il nemico politico della Chiesa Cattolica o del papa, poiché:

“Nella Divina Commedia di Dante il genio del poeta dispose in un'impressionante interezza ciò che allora era vivo nelle tradizioni popolari ed ecclesiastiche, Giotto e Dante attinsero a un'unica fonte senza imitarsi a vicenda, servendosi di un retaggio universale. Da qui ovviamente anche la loro somiglianza". Kraszewski infatti non credeva che Dante avesse potuto influenzare Giotto. Questa supposizione dello scrittore è oggi avallata dalla critica che considera una loro probabile amicizia, nonché una loro collaborazione, semplicemente come qualcosa di leggendario, dacché essa non è supportata da nessuna documentazione scritta [cfr. De Carlo 2008: 189]. Non si dimentichi che essi non condivisero né ideali politici né convinzioni religiose: ad esempio Dante era nemico di quasi tutti i committenti di Giotto, specialmente di papa Bonifacio viII. Non si può però escludere un loro incontro a Padova, dove Dante si trovava in esilio, mentre Giotto affrescava la Cappella degli Scrovegni: è verosimile che in quel periodo ci sia stato fra i due uno scambio di idee e di opinioni [cfr. Zeri 1998: 17]. 
Świat ten czyścowy to już jakby drugiéj epoki żywota ludzkości wizerunek; w nim człowiek uczuwa w sobie boską iskierkę, pochodzenie swe [...]; wie, że musi walczyć i dobijać się, aby duchem zwyciężając ciało do boskiego przybliżył się wzoru i z Bogiem miał prawo się połączyć” [Kraszewski 1869a: 145]. ${ }^{28}$

Per Kraszewski, dunque, in ogni cantica della Divina Commedia si può cogliere una determinata caratteristica: "W Piekle panowała namiętność, w Czyścu znajdujemy uczucie, w Raju czystego ducha. Czyściec jest obrazem ziemi, na któréj walczym, nieustannie się oczyszczając, podnosząc, uszlachetniając" [Kraszewski 1869a: 145]. ${ }^{29}$ Lo scrittore probabilmente attinge alle idee enunciate da Friedrich Schelling (1775-1854), che nei suoi scritti filosofici aveva osservato: l'Inferno è plastico, scultoreo, il Purgatorio dipinto, disegnato, il Paradiso è musicale perché è un canto, una melodia. Kraszewski, riverberando le teorie di Ozanam, che pure Julian Klaczko aveva rinnovato nelle sue famose Causeries Florentines, sostiene che il Purgatorio costituisce la cantica più gradevole di tutto il poema: qui l'uomo incontra creature simili a lui, più vicine all'esistenza terrena [Frąckiewicz 1912: 37-38]. Se negli inferi Dante soffre per la disperazione dei dannati, nel purgatorio scorge persone con la speranza di espiare i propri peccati.

Nella parte dedicata al Purgatorio Kraszewski chiarisce che l'Inferno è sempre stata la cantica che ha affascinato di più i suoi contemporanei: "Dante jest głównie znany jako malarz piekła, dla nie wielu jest wieszczem niebios” [Kraszewski 1869a: 132] .30 Il critico polacco individua la causa principale nel fatto che la prima cantica fosse più accessibile alla comprensione del lettore medio; anche se, sempre secondo l'autore, il Purgatorio e il Paradiso non hanno

"Il mondo purgatoriale è già come se fosse la vita di una seconda epoca dell'umanità; in esso l'uomo sente in sé il barlume divino, le sue origini [...]; sa che deve lottare e ottenere, affinché vincendo collo spirito il corpo, possa avvicinarsi al modello divino e abbia il diritto di ricongiungersi con Dio."

"Nell'Inferno domina la passione, nel Purgatorio il sentimento, nel Paradiso il puro spirito".

30 "Dante è principalmente conosciuto come il maestro dell'inferno, per pochi è il vate dei cieli." 
niente da invidiare all'Inferno per bellezza ed esecuzione. Inoltre, a differenza dell'Inferno e del Paradiso, la seconda cantica attinge meno alle leggende, ed è strettamente connessa con l'immagine della terra, con le sue lotte che purificano l'anima e la nobilitano. Nel Purgatorio vi sono meno personaggi e tutto si concentra sulla personalità del poeta. Kraszewski si immerge insieme all’Alighieri in questo nuovo mondo: "Czyściec najbliższy życia i ziemi [...], ma niezrównany wdzięk wyidealizowanego świata naszego i naszego żywota." [Kraszewski 1869a: 147]..$^{31}$ L'autore più avanti chiarisce: "W Czyścu współczuciem łączemy się bliżej z istotami zaludniającemi go, które mają słabości nasze, w których poznajemy samych siebie - choć podniesionych do ideału" [Kraszewski 1869a: 147].$^{32}$ Kraszewski dissente ancora con quei commentatori che nella simbologia del poeta hanno intravisto il mistero totale. Sebbene la Commedia sia allegorica, tuttavia - afferma Kraszewski - non è misteriosa [Kraszewski 1869a: 162]. In più egli polemizza con il Rossetti e tutti coloro che discersero un tono apocalittico nella visione di Dante [Gorskij 1968: 47].

L'autore contrasta anche le opinioni di tutti quei commentatori che hanno visto in Dante il nemico del papa e del Vaticano. L'Alighieri non fu mai un avversario del papato, egli rimproverava solo alla Santa Sede il suo interesse per il potere temporale e le ricchezze materiali [Gorskij 1968: 48]. La Chiesa, invece di perseguire il fine della salvezza delle anime, pensava solo alla potenza terrena, cercando di sostituirsi all'imperatore, ma così facendo, accresceva solo il caos, e al tempo stesso si corrompeva nella ricerca dei beni mondani; l'assenza di ogni controllo dall'alto fa sì che si scateni la cupidigia di denaro, nasca negli uomini la volontà di sopraffarsi a vicenda, e di qui iniziano i conflitti tra le fazioni e le infinite lotte civili che insanguinavano le città italiane. Dante si oppose a questo stato di cose, agendo per l'interesse della patria e i principî di giustizia. In questo lo scrittore polacco si sentiva spiritualmente molto

"Il Purgatorio è più affine alla vita e al mondo terreno [... ], ha la bellezza eccezionale del nostro pianeta idealizzato e della nostra vita."

32 "Nel Purgatorio attraverso la compassione ci uniamo più strettamente alle creature che lo popolano, che hanno le nostre debolezze, in esse riconosciamo noi stessi, anche se idealizzati." 
vicino al poeta fiorentino, proprio per la medesima condizione in cui si ritrovò quando venne bollato di anticlericalismo.

Il "Titano del lavoro" 33 dimostrò che le classi dominanti traviano il pensiero cristiano, autentico e primigenio. Questa opinione chiarisce a prima vista il fatto paradossale che Kraszewski abbia acquisito cattiva fama per la sua lotta intransigente con la reazione clericale; però nelle lezioni su Dante esalta il Cristianesimo, lo idealizza, addirittura gli attribuisce lo sviluppo dell'intera umanità. Per cui quando egli chiama Dante l' "altissimo poeta", ha in mente proprio quest'idea: l'autore della Commedia non solo diede in essa la più geniale sintesi medievale, sebbene, d'altra parte, avesse cancellato lo iato esistente tra Medioevo e Rinascimento, ma aprì anche una nuova epoca nello sviluppo spirituale dell'uomo [Gorskij 1968: 39]. Kraszewski, riprendendo il giudizio di Giambattista Vico, arguisce che, come l'Iliade, la Divina Commedia rappresenta un eccezionale riepilogo della sua epoca [Gorskij 1968: 38 ]:

Żadnemu może z wielkich poetów, przedstawicieli przeszłości, słuszniéj się nie należy jak Dantemu to nadużyte określenie że sobą całą uosabia epokę; - żaden w istocie, prócz Homera, nie wcielił tak swego wieku, jego charakteru, jenijuszu i wiedzy, jak wielki wieszcz florencki - l'altissimo poeta. Żaden też z chrześciańskich poetów nowéj ery nie jest nadeń bardziéj chrześciańskim, wybitniéj synem tego świata, który na gruzach pokruszonéj starożytności się wznosi [Kraszewski 1869a: 97].34

La conclusione delle lezioni di Krazewski è un atto di riverenza nei confronti del poeta fiorentino al quale deve molto tutto il mondo civile [Morawski 1965: 96]. Secondo lo scrittore, la Divina Commedia è nata in un'epoca in cui molte verità non erano state a Dante la consunta espressione: egli incarna tutta la sua epoca; nessuno infatti, eccetto Omero, è divenuto il simbolo del proprio secolo, della sua peculiarità, del suo genio e del suo sapere, quanto il grande vate fiorentino, l'altissimo poeta. Nessun poeta cristiano della nuova era è stato più cristiano di lui, più figlio di questo mondo che s'innalza sulle rovine dell'antichità sgretolata." 
ancora rivelate e le leggende detenevano un ruolo primario. I sentimenti e i concetti espressi nella Commedia non possono più essere ripetuti, come per esempio l'opera di Beato Angelico non può essere più riprodotta [Morawski 1965: 96]. Pertanto, il poema dantesco è irripetibile e immortale, e il suo eccezionale valore, come scrisse Kraszewski, si coglie soprattutto nelle piccole cose, alla pari della natura che è, secondo un proverbio latino, "maxima miranda in minimis" [Kraszewski 1869: 143].

\section{Kraszewski dilettante?}

Le lezioni sulla Commedia del 1867, preparate dallo scrittore per le istituzioni di Cracovia e di Leopoli, dunque, costituiscono indubbiamente un notevole apporto alla diffusione di Dante in patria. Ciò contribuì ad ascrivere Kraszewski nel novero dei dantisti polacchi ed europei: l'eminente filologo romanzo Kalikst Morawski sottolineò, infatti, che agli albori della dantistica polacca, per quel che concerne i primi esperti "scientifici", si può includere, accanto a Julian Klaczko, altresì Kraszewski.

Non mancarono tuttavia critiche agli Studia da parte dei connazionali e contemporanei di Kraszewski; tra di essi, in prima linea, vi fu Kazimierz Bogusławski. Quest'ultimo nel suo ampio saggio Dante $w$ Polsce (Dante in Polonia), pur considerando interessanti gli studi di Kraszewski, li reputa privi di attitudine scientifica: critica la mancanza di metodo e qualifica lo scrittore come dilettante. Il critico dissente fortemente col saggio kraszewskiano, bollandolo di superficialità, poiché egli ha trascurato, dopo i lavori di Ozanam, Fauriel, Witte, Philalethes, Wegele, Blanc e Scartazzini, i più importanti momenti della vita di Dante che hanno così profondamente agito sulla sua formazione e nutrito il suo genio. Bogusławski lamentò ancora la scarsa precisione nell'esporre il periodo storico in cui visse il poeta; il non aver approfondito quei principî che fecero nascere una forza creativa così potente e prodiga di emozioni; l'assenza di indicazioni scientifiche che servono a conferire una visione uniforme e armonica al lettore. Inoltre il critico nota la mancata trattazione di argomenti che sono alla base del grande poema: la poesia dell'amore, il culto della donna, il significato di Beatrice 
e di Virgilio, la parte teologica, filosofica, politica, cosmogonica, storica, astronomica e via dicendo [Bogusławski 1885: 747]. In breve egli fa un'ampia analisi delle lacune di Kraszewski, confrontandolo con i grandi dantisti dell'epoca. A titolo di esemplificazione si può citare il seguente frammento:

Podobnie jak końcowe pieśni Raju, lub słynną rozmowę Cacciaguidy, traktuje Kraszewski spotkanie Danta z Matyldą. [ ... ]

Autor nie podaje nawet, kimby ta postać była: czy osobą rzeczywistą z otoczenia poety, czy personifikacyą jakiej idei, czy postacią alegoryczną, czy z dziejów wziętą? A przecież w przedmiocie tym pisano nie mało. Podawano różniej czasy aż siedm osób historycznych, które chciano widzieć pod tą postacią. Było też kilkanaście interpretacyj alegorycznych. [Bogusławski 1885: 752 ]. ${ }^{35}$

Il bibliotecario e cultore di lingue e letterature romanze Walerian Preisner, invece, considera il saggio di Kraszewski, pur riconoscendone una serie di punti deboli e di mancanze metodologiche, non del tutto "superficiale", né tanto meno dilettantesco [Preisner 1957: 57]. In generale si può aggiungere che le critiche mosse a Kraszewski da Bogusławski restano discutibili, poiché, al contrario, lo scrittore dimostrò di avere una conoscenza di Dante piuttosto approfondita per l'epoca. Le conferenze dell'autore di Stara baśń, infatti, sono da valutare in relazione ai tempi: tutte le inesattezze riportate da Kraszewski sono da imputare alle interpretazioni del poema dantesco che circolavano in quel periodo e agli imprecisi strumenti e alle limitate informazioni di cui lo stesso studioso poteva disporre. Le lezioni kraszewskiane, malgrado si dimostrino inadeguate in alcuni luoghi, avevano però - ribadiamo - il merito

"Kraszewski tratta l'incontro di Dante con Matelda parimenti ai canti finali del Paradiso, o il famoso dialogo di Cacciaguida. [... ] tuttavia l'autore non indica chi sia questa figura: una persona reale della cerchia del poeta, la personificazione di qualche idea, una figura allegorica o presa dalla storia? Eppure su questo argomento non è stato scritto poco. Nei momenti più diversi sono stati indicati fino a sette personaggi storici nei quali si voleva ravvisare questa figura. Vi sono state anche una decina di interpretazioni allegoriche." 
di divulgare Dante in Polonia [Preisner 1957: 55]; infatti, i tentativi interpretativi e le notizie biografiche rappresentavano un incentivo alla lettura, nonché alla diffusione presso una larga parte di pubblico della Commedia.

Olga Płaszczewska osserva che l'intento delle lezioni di Kraszewski - come era consuetudine all'epoca dal Towarzystwo Wzajemnej Pomocy Uczniów Uniwersytetu Jagiellońskiego [cfr. Frąckiewicz 1912: 7-8] - era prettamente divulgativo. Secondo la studiosa, l'autore in questa occasione indossa la "maska dyletanta" (maschera del dilettante) quale strategia che gli consente di avere maggiore libertà interpretativa e di scelta rispetto alle tradizionali conferenze accademiche [Płaszczewska 2012: 193]. Lo scrittore conosceva bene l'Italia e la lingua italiana [cfr. De Carlo 2007], era stato un appassionato lettore della Commedia e nella sua biblioteca si trovavano diverse edizione del poema e numerosi commenti in italiano, francese, inglese e tedesco [cfr. Pawlik 1888: 407-409; Wasyłenko 1993: 20-21], continui sono anche i riferimenti alla Divina Commedia nelle sue opere [cfr. Piekut: 31-39; De Carlo 2016: 39-44], recitava a memoria alcuni frammenti e dipingeva scene del poema [Frąckiewicz 1912: 15-16; Trepiński 1970: 54; Płaszczewska 2012: 193-194; 2017: 41-42].

Alla luce di quanto asserito, dunque, si può affermare senza il timore di incorrere in eccessive semplificazioni né in facili conclusioni che gli Studja nad Komedją Boską di Kraszewski, malgrado le mancanze e le ipotesi ormai ampiamente confutate dalla critica, restano non solo un contributo significativo alla divulgazione del poeta italiano e della Divina Commedia in Polonia, ma anche rispetto ai tempi un valido apporto al progresso e allo sviluppo della dantistica polacca.

\section{Bibliografia}

Alighieri Dante (1870), Komedya Boska. - Czyściec, illustracye G. Dorèego, objaśnienia J.I. Kraszewskiego, “Tygodnik Ilustrowany”, n. 156, vol. vi, pp. 307-310.

Alighieri Dante (1875), Czyściec - Pieśń XI. Przekład J.I. Kraszewskiego, in: Sobótka. Księga zbiorowa na uczczenie pięćdziesięcioletniego jubileuszu 
Seweryna Goszczyńskiego, Przedpłaciciele, W komisie księgarni Gubrynowicza i Schmidta, Lwów, pp. 127-131.

Alighieri Dante (1909), Boska komedia, tłum. E. Porębowicz, Gebethner i Wolff, Warszawa.

Bachórz Józef (1990), Zdziwienia Kraszewskim, in: Zdziwienia Kraszewskim, pod red. Marty Zielińskiej, Zakład Narodowy im. Ossolińskich, Wydawnictwo PAN, Wrocław-Warszawa-Kraków, pp. 139-155.

Biliński Bronisław (1965), Roma antica e moderna nelle opere di Giuseppe Ignazio Kraszewski, Wydawnictwo PAN, Wrocław-Warszawa-Kraków. Biliński Bronisław (1971), Incontri polacco-italiani a Porta Pia:

J.I. Kraszewski, W. Kulczycki, M. Konopnicka. Nel centenario di Roma capitale d'Italia 1870-1970, Wydawnictwo PAN, Wrocław-WarszawaKraków-Gdańsk.

Bogusławski Kazimierz (1885), Dante w Polsce, "Niwa”, fasc. 260-261, 625-635, 746-770.

Boito Arrigo (2008), Il libro dei versi, a cura di Claudio Mariotti, Mucchi Editore, Modena.

Bursztyńska Halina (1998), Kraszewski Orzeszkowa Sienkiewicz. Studia i szkice, Wydawnictwo Naukowe wsp, Kraków.

Ciampoli Domenico (1891), Giuseppe Ignazio Kraszewski, in: Id., Studi letterari, Niccolò Giannotta Editore, Catania, pp. 401-415.

Dąbrowolska Maria Halina (1855), Tytan pracy. Opowieść o Józefie Kraszewskim, Ludowa Spółdzielnia Wydawnicza, Warszawa.

De Carlo Andrea Fernando (2007), Alla ricerca dell'Arcadia degli artisti: il viaggio in Italia di Józef Ignacy Kraszewski, "Studia Romanica Posnaniensia”, vol. xxxiv, pp. 151-165.

De Carlo Andrea Fernando (2008), Dramat w niebie i na ziemi. Kraszewski jako krytyk Giotta w „Kartkach z podróży 1858-1864”, in: Drogi i bezdroża komunikacji, pod red. Piotra Beringa, Grzegorza Łukomskiego, Wydawnictwo Kropka J. i W. Śliwczyńscy, Gniezno, pp. 185-195.

De Carlo Andrea Fernando (2014), O specyfice języka J.I. Kraszewskiego w rękopisie zawierajacym ttumaczenie „Boskiej Komedii” Dantego, in: Kraszewski i wiek XIX. Studia, idea i układ tomu Jarosław Ławski, red. Anna Janicka, Krzysztof Czajkowski, Paweł Kuciński, Książnica Podlaska im. Łukasza Górnickiego, Katedra Badań Filologicznych „Wschód-Zachód”, Wydział Filologiczny Uniwersytetu w Białymstoku, Białystok, pp. 291-309.

De Carlo Andrea Fernando (2016), Między piektem a niebem. Postrzeganie „Boskiej Komedii” Dantego przez Zygmunta Krasińskiego i Józefa Ignacego Kraszewskiego, in: Krasiński i Kraszewski wobec europejskiego 
romantyzmu i dylematów XIX wieku. $W$ dwustulecie urodzin pisarzy, red. M. Junkiert, W. Ratajczak, T. Sobieraj, Wydawnictwo Poznańskiego Towarzystwa Przyjaciół Nauk, Poznań 2016, pp. 33-45.

De Carlo Andrea Fernando (2017), "La Divina Commedia" nella Polonia del XIX secolo. Le prime traduzioni polacche del poema dantesco a confronto, in: Il Dante dei moderni. La Commedia dall'Ottocento a oggi. Saggi critici, a cura di Joanna Szymanowska, Izabela Napiórkowska, LoGisma, Firenze, pp. 125-144.

De Carlo Andrea Fernando (2019a), Dantes in miranda in minimis. Kraszewski e Dante, Orientalia Parthenopea Edizioni, Napoli.

De Carlo Andrea Fernando (2019b), «la dove 'l sol tace». La complessità metaforico-sinestetica della Divina commedia e la sua resa nelle prime traduzioni polacche: Korsak, Kraszewski, Stanisławski e Porębowicz, in: «Vedi lo sol che ' $n$ fronte ti riluce»: La vista e gli altri sensi in Dante e nella ricezione artistico-letteraria delle sue opere, a cura di Maria Maślanka-Soro, con la collaborazione di Anna Pifko-Wadowska, Aracne, Roma, pp. 197-210.

De Carlo Andrea Fernando (2019c), "Honor the great poet". The Józef Ignacy Kraszewski's contribution to the development of Polish Dante studies, “Acta Universitatis Lodziensis. Folia Litteraria Polonica”, n. 4 (55), pp. 363-381.

Finazzi Silvia (2008), La metafora scientifica e la rappresentazione della Corporeitas luminosa, in: La metafora in Dante, a cura di Marco Ariani, Leo S. Olschki Editore, Firenze, pp. 167-192.

Fiorentino Carlo M. (2003), Un esule polacco in Italia. Wtadisław [sic!] Sas Kulczycki (1831-1895), Archivio Guido Izzi, Roma.

Frąckiewicz Michał (1912), Józef Ignacy Kraszewski w Krakowie i we Lwowie w r. 1867 i odczyty jego o Dantem. Wspomnienia z przeszłości w setna rocznicę urodzin, Nakładem Autora, Kraków.

Gorskij Ivan K. (1968), Крашевский и Аанте, “Аантовские чтения”, pp. 33-52.

Klaczko Julian (1904), Szkice i rozprawy literackie, z upoważnienia autora tłumaczyli Stanisław Tarnowski, Józef Jabłonowski i Antoni Potocki, z przemową Stanisława Tarnowskiego, Nakładem Kięgarni F. Hoesicka, Warszawa.

Klaczko Julian (1925), Conversazioni fiorentine. Dante e Michelangelo. Beatrice e la poesia d'amore, Dante e il cattolicismo, la tragedia di Dante, traduzione di Giovanni Sanna, Laterza e figli, Bari.

Kraszewski Józef Ignacy (1866a), Kartki z podróży 1858-1864 r. - Kraków, Wiedeń, Triest, Wenecja, Padwa, Medjolan, Genua, Piza, Florencja, Rzym, vol. I, Nakładem Gustawa Sennewalda, Warszawa. 
Kraszewski Józef Ignacy (1866b), Trzy ostatnie pieśni Danta Komedyi Boskiej: przekład Kraszewskiego, "Biblioteka Warszawska”, vol. I, pp. 389-398.

Kraszewski Józef Ignacy (1869a), Dante. Studja nad Komedją Bozka przez J.I. Kraszewskiego, "Roczniki Towarzystwa Przyjaciół Nauk Poznańskiego”, vol. v, pp. 95-189.

Kraszewski Józef Ignacy (1869b), Dante (z odczytów J.I. Kraszewskiego 1867 r.), in: Kłosy i kwiaty. Książka zbiorowa w Krakowie, W Drukarni W. Kirchmayera, Kraków, pp. 67-97.

Kraszewski Józef Ignacy (1870), Dante. Vorlesungen ueber die Goettliche Komoedie Gehalten in Krakau und Lemberg 1867 von J.I. Kraszewski, Ins deutsche uebertragen von S. Bohdanowicz, Druk und Verlag von J.I. Kraszewski, Dresden.

Kraszewski Józef Ignacy (1972), Pamiętniki, oprac. Wincenty Danek, Zakład Narodowy im. Ossolińskich, Wrocław.

Kraszewski Józef Ignacy (1993), Listy do rodziny 1863-1886, część II (Na emigracji), oprac. Stanisław Burkot, Zakład Narodowy im. Ossolińskich, Wrocław - Warszawa - Kraków.

Litwornia Andrzej (2005), „Dantego któż się odważy tłumaczyć?”. Studia o recepcji Dantego $w$ Polsce, IBL, Warszawa.

Łempicki Stanisław (1930a), Dante i kultura włoska w Polsce (dwa odczyty), osobne odbicie z Feljetonów „Gazety Lwowskiej”Odbito w Drukarni Polskiej we Lwowie, Lwów.

Łempicki Stanisław (193ob), Dante a Polska, in: Towarzystwo „Dante Alighieri” we Lwowie (upamiętnienie inauguracji), Czcionkami „Drukarni Polskiej” we Lwowie, Lwów, pp. 29-65.

Maślanska-Soro Maria (2015), Antyczna tradycja epicka u Dantego, Księgarnia Akademicka, Kraków.

Maślanska-Soro Maria (2004), La persuasion émotive dans «L'Enfer» de Dante, in: Le cahiers des paralittératures 8 (Traduire l'émotion), études réunies par Jan Herman, Nathalie Kremer, Marcela Swiątkowska, Cefal, Liège, pp. 79-90.

Maślanska-Soro Maria (2004), Posłowie [Note al testo], in: Dante Alighieri, Boska Komedia, przełożył Edward Porębowicz, Wydawnictwo Zielona Sowa, Kraków, pp. 467-476.

Maślanska-Soro Maria (2000), Semantyka przestrzeni w Pieśni IV „Piekła” Dantego, in: Prace Komisji Neofilologicznej PAU, vol. I, pod red. Olgi Dobijanki-Witczakowej, Stanisława Widłaka, PAU, Kraków, pp. 61-76. Maślanska-Soro Maria (2017), Traduzione come interpretazione: nella Commedia e della Commedia, in: Il Dante dei moderni. La Commedia dall'Ottocento a oggi. Saggi critici, a cura di Joanna Szymanowska, Izabela Napiórkowska, LoGisma, Firenze, pp. 157-166. 
Maślanska-Soro, Maria (2005), Tragizm w „Komedii” Dantego, Universitas, Kraków.

Matuszewski Ignacy (1899), Dyabet w poezyi. Historya i psychologia postaci uosabiających zło w literaturze pięknej wszystkich narodów i wieków, B. Natason, Warszawa 1899, pp. 93-97.

Miszalska Jadwiga et al. (2007), Od Dantego do Fo. Wtoska poezja $i$ dramat w Polsce (od XVI do XXI wieku), Collegium Columbinum, Kraków.

Morawski Kalikst (1965), La dantologia polacca moderna, "Sonderdruck aus Beitræge zur Romanischen Philologie”, fasc. 2 (IV), pp. 90-91.

Pawlik Michał (1888), Danteana, in: Katalog: księgozbioru, rękopisów, dyplomów, rycin, map, atlasów, fotografii, jako też osobistych dyplomów, adresów itp. pozostatych po śp. Józefie Ignacym Kraszewskim staraniem Franciszka Kraszewskiego, uporządkował i spisał Michał Pawlik, „Drukarnia Polska”, Lwów, pp. 407-409.

Piekut Stanisław (1967), Tematyka włoska w twórczości J.I. Kraszewskiego, "Oficyna Poetów", n. 3 (6), pp. 31-38.

Płaszczewska Olga (2012), „Onorate l'altissimo poeta”: Józef Ignacy Kraszewski jako komentator Dantego, in: Kraszewski: poeta $i$ światy, red. Tadeusz Budrewicz, Ewa Ihnatowicz, Ewa Owczarz, Wydawnictwo Naukowe Uniwersytetu Mikołaja Kopernika, Toruń, pp. 191-203.

Płaszczewska Olga (2017), I. Z polskich odczytań klasyki włoskiej: Józef Ignacy Kraszewski jako komentator Dantego, in: Ead., Wtoskie divertimento. Szkice komparatystyczne, Wydawnictwo UJ, Kraków, pp. 37-54. Preisner Walerian (1957), Dante i jego dzieła w Polsce. Bibliografia krytyczna z historycznym wstępem: I. Stan badań nad Dantem $w$ Polsce; II. Próba polskiej bibliografii dantejskiej / Dante e le sue opere in Polonia. Bibliografia critica con una introduzione storica: I. Gli studi danteschi in Polonia; II. Saggio d'una bibliografia dantesca polacca, PWN, Toruń.

Scartazzini Andrea Giovanni (1871), [rec.] Dante, Vorlesungen ueber die Goettliche Komoedie, gehalten in Krakau und Lemberg 1867 von J.I. Kraszewski. Ins deutsche uebertragen von S. Bohdanowicz, Dresden Druk und Verlag von J.I. Kraszewski, 1870, "Nuova Antologia”, vol. 17, pp. 524-526.

Scartazzini Andrea Giovanni (1881), Dante in Germania. Storia letteraria e bibliografica dantesca alemanna. Dal secolo XIV sino ai nostri giorni, vol. 1, U. Hoepli, Napoli-Milano-Pisa, pp. 203-205.

Stanislavskij Antoni (1864), Аанте Алигиери. Биографический очерк читаньй на литературном вечере 5 апреля 1864 2., в пользу Мариинского женского училища в Харькове, Санкт-Петербург. 
Trepiński Antoni (1970), Romans Kraszewskiego z Wiedenka, PIW, Kraków.

Vallone Aldo (1975), La critica dantesca nell'Ottocento, Leo S. Olschki Editore, Firenze.

Wasyłenko Wołodymyr (1993), Polskie losy Dantego w wieku XIX. Prolegomena do „zaginionego" ttumaczenia „Boskiej Komedii” dokonanego przez J.I. Kraszewskiego, Wydawnictwo PTPN, Poznań. Zeri Federico (1998), Giotto. Compianto sul Cristo morto, Rizzoli, Milano.

\section{Andrea F. De Carlo}

\section{Studies on The Divine Comedy by Józef Ignacy Kraszewski and the} Beginnings of Polish Dante Studies (1820-1870)

The article analyses Józef Ignacy Kraszewski's contribution to the development of Polish Dante studies - thanks to his lectures on Dante held in Krakow and Lviv in 1867 and later published under the title Studies on The Divine Comedy, 1869. The article presents other opinions of Dante scholars of the time and more or less critical reviews of the results of Kraszewski's research.

Keywords: Polish Dante studies; Józef Ignacy Kraszewski; Dante Alighieri; Studies on The Divine Comedy.

Andrea F. De Carlo - professore a contratto di Lingua e Letteratura Polacca presso l'Università degli Studi di Napoli “L'Orientale” e l'Università degli Studi di Bari "Aldo Moro". I suoi ambiti di ricerca comprendono la letteratura polacca, i rapporti culturali fra Italia e Polonia e la traduzione poetica. I risultati delle sue ricerche sono pubblicati in diverse riviste polacche e italiane. Ha pubblicato vari articoli su Sienkiewicz, Kraszewski, Leśmian e Kapuściński ed è autore della monografia Dantes maxime mirandus in minimis. Kraszewski e Dante, Orientalia Parthenopea Edizioni, Napoli 2019. Attualmente lavora all'edizione critica della traduzione polacca della Divina Commedia a opera di J.I. Kraszewski. Contatto: afdecarlo@unior.it; andreafernando.decarlo@ uniba.it. 